\title{
RS3PE syndrome as a rare differential diagnosis in edema of the upper extremity. Presentation of two patients with edema refractory to de-resuscitation after volume overload in sepsis
}

\author{
H. Strawe, T. Schilling, D. Raepple
}

Internal Medicine Intensive Care, Katharinen Hospital, Klinikum Stuttgart, Germany

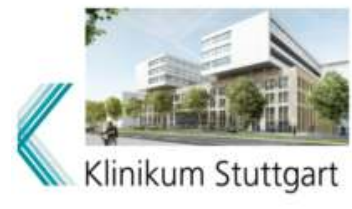

Background. In 1985, McCarty et al. described patients (pts) with an abrupt beginning of bilateral synovitis with pitting edema. The syndrome was named "Remitting Seronegative Symmetrical Synovitis with Pitting Edema", RS3PE. Further magnetic resonance imaging (MRI) and ultrasound imaging (US) studies showed extensor tendosynovitis edema of the peritendinous and subcutaneous soft tissue. There is a male predominance $(2: 1)$ and elderly pts are mainly affected. The serological workup in the majority of these patients showed negativity for rheumatoid factor. An association could be demonstrated with polymyalgia rheumatica (PMR), rheumatoid arthritis (RA) and many other rheumatic diseases. Paraneoplastic RS3PE has also been described and lately two case reports were published of RS3PE after nivolumab. Common to all is a dramatic response to corticosteroids and an good prognosis.

Objectives. In sepsis, various mechanisms contribute to endothelial injury and capillary leakage. High volume fluid resuscitation often leads to the formation of generalized edema. De-resuscitation is a major challenge. Persisting peripheral edema despite negative volume balance should raise suspicion for co-existing problems like severe hypalbuminemia, thrombosis or myxedema. By presenting two cases of persisting edema we want to draw attention to a rare differential diagnosis, RS3PE.

Material and Methods. Informed consent was obtained from the legal substitute. Ultrasound studies were performed with a Philips(R) CX-50.

Case Presentation 1. A 76-year-old male pt was transferred our ICU. After surgery he suffered septic multiple organ failure caused pneumonia (HAP). Previously known were alcohol abuse and hypothyroidism. Despite de-resuscitation of persisting volume overload, bilateral edema of the hands persisted. Thromboses of the upper extremities were excluded, thyroid hormones were in normal range. Retrospectively, the diagnosis of pre-existing PMR was based on a family member reporting extreme bilateral painfulness in the shoulder area in the weeks before. There was a markedly increased blood sedimentation rate. Rheumatoid factor was unspecific, antibodies against cyclic citrullinated peptide (CCP) were negative. On corticosteroids, a rapid improvement of the edema and of PMR symptoms resulted.
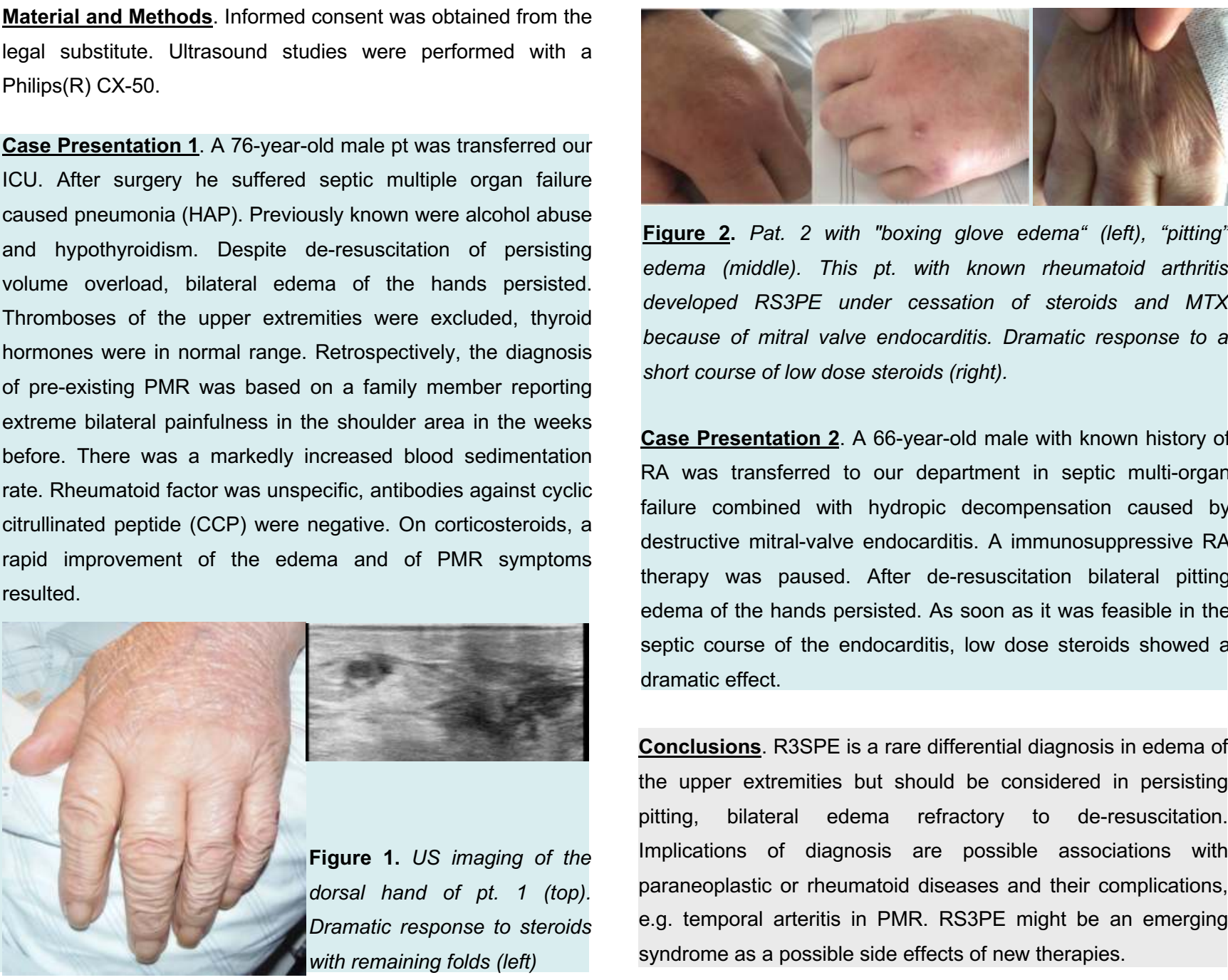

Figure 2. Pat. 2 with "boxing glove edema" (left), "pitting" edema (middle). This pt. with known rheumatoid arthritis developed RS3PE under cessation of steroids and MTX because of mitral valve endocarditis. Dramatic response to a short course of low dose steroids (right).

Case Presentation 2. A 66-year-old male with known history of RA was transferred to our department in septic multi-organ failure combined with hydropic decompensation caused by destructive mitral-valve endocarditis. A immunosuppressive RA therapy was paused. After de-resuscitation bilateral pitting edema of the hands persisted. As soon as it was feasible in the septic course of the endocarditis, low dose steroids showed a dramatic effect.

Conclusions. R3SPE is a rare differential diagnosis in edema of the upper extremities but should be considered in persisting pitting, bilateral edema refractory to de-resuscitation. Implications of diagnosis are possible associations with paraneoplastic or rheumatoid diseases and their complications, e.g. temporal arteritis in PMR. RS3PE might be an emerging syndrome as a possible side effects of new therapies.

References

1.McCarty DJ et. al. . Remitting seronegative symmetrical synovitis with pitting edema. RS3PE syndrome. JAMA. 1985;254(19):2763-2767.

2.Agarwal V et. al. Remitting seronegative symmetrical synovitis with pitting edema (RS3PE) syndrome: ultrasonography as a diagnostic tool. Clin Rheumatol. 2005;24(5):476-479.

3.Olivieri I et. al. RS3PE syndrome: an overview. Clin Exp Rheumatol. 2000;18(4 Suppl 20):S53-S55.

4.Gauci M-L et al. Remitting seronegative symmetrical synovitis with pitting edema (RS3PE) syndrome induced by Nivolumab. Seminars in Arthritis and Rheumatism. March 2017:1-19. 